October 2016

\title{
Therapeutic communication and relationships in chronic and complex care
}

Sharon Brownie

Aga Khan University, sharon.brownie@aku.edu

Robin Scott

Charles Sturt University

Rachel Rossiter

Charles Sturt University

Follow this and additional works at: http://ecommons.aku.edu/eastafrica_fhs_sonam

Part of the Nursing Commons

\section{Recommended Citation}

Brownie, S., Scott, R., Rossiter, R. (2016). Therapeutic communication and relationships in chronic and complex care. Nursing Standard, 31(6), 54-63.

Available at: http://ecommons.aku.edu/eastafrica_fhs_sonam/88 


\title{
Therapeutic communication and relationships in chronic and complex care
}

NS864 Brownie S et al (2016) Therapeutic communication and relationships in chronic and complex care. Nursing Standard. 31, 6, 54-6I. Date of submission: 10 December 2014; date of acceptance: 5 August 20l6. doi: 10.7748/ns.2016.e9847

\section{Sharon Brownie Dean, School of Nursing and Midwifery, Aga Khan University, East Africa \\ Robin Scott \\ Teaching academic, School of Nursing, Midwifery and Indigenous Health, Faculty of Science, Charles Sturt University, New South Wales, Australia \\ Rachel Rossiter Associate professor of nursing and nurse practitioner, School of Nursing, Midwifery and Indigenous Health, Faculty of Science, Charles Sturt University, New South Wales, Australia}

\section{Correspondence \\ s.brownie@griffith.edu.au}

Conflict of interest None declared

\section{Peer review}

This article has been subject to external double-blind peer review and checked for plagiarism using automated software

\begin{abstract}
As the population ages and the incidence of chronic diseases and lifestyle-related conditions rises, nurses are increasingly required to provide care for people with a range of chronic (long-term) conditions. The healthcare needs of patients are often complicated by comorbid conditions. Nurses deliver healthcare in the context of the patient's medical conditions, treatment regimens, the healthcare system, and the individual's socioeconomic, personal and family factors, which may include the challenges of social isolation and geographic distance. In such complex circumstances, patients may be perceived as 'difficult' or 'challenging', however, the challenge is not the patient themselves, but the relationship between the nurse and the patient. Communication difficulties can occur between nurses and patients, which may affect the therapeutic relationship and the quality of care provided. This article discusses the communication skills that nurses require to interact effectively with patients who have complex and chronic comorbid conditions. It focuses on therapeutic communication strategies and the nurse-patient relationship, while emphasising the need for nurses to be self-aware when caring for patients with complex healthcare needs.
\end{abstract}

\section{Keywords}

chronic care, comorbidities, complex care, long-term conditions, nurse-patient relationship, therapeutic communication, therapeutic relationship

\section{Aims and intended learning outcomes}

This article aims to enhance awareness and understanding of the therapeutic communication skills that nurses require to deliver effective care for patients with complex and chronic conditions. After reading the article and completing the time out activities you should be able to:

"Understand the concepts of complexity and chronicity in relation to healthcare, and the factors that may contribute to them.

"Outline the therapeutic communication skills necessary to communicate effectively with people who have complex and chronic comorbid conditions.
» Recognise factors that might adversely affect the therapeutic relationship between nurses and patients.

"Identify personal factors that might reduce your ability to communicate effectively as a healthcare professional.

" Review your current strategies in communicating with patients who have complex and chronic comorbid conditions, particularly those who are perceived as 'challenging'.

"Consider ways to achieve a balance between caring for others and caring for yourself in your clinical practice.

\section{Introduction}

Chronicity, complexity, comorbidity and multimorbidity are terms that are 
increasingly used in healthcare and research settings. Therefore, it is important for healthcare professionals to understand what is meant by these terms and to consider why complex and chronic conditions are increasing, and the implications of this for nursing. Definitions of these commonly used terms are provided in Box 1.

There are several factors that have contributed to the global shift from healthcare presentations dominated by acute problems to healthcare presentations characterised by complexity and chronicity (Ministry of Health 2016). For example, although people are living longer, they are experiencing higher levels of disability. On average, life expectancy has increased by more than 10 years for men and women; however, in these later years, people often experience chronic pain, physical disability and cognitive impairments (Nolte et al 2008, Ministry of Health 2016). Simultaneously, the lifestyle behaviours of a significant proportion of the population have changed, with increasing inactivity; high intake of sugar and processed food; low intake of fresh fruit and vegetables; excessive salt intake; and continuing high rates of smoking and alcohol misuse (Ministry of Health 2016).
Lifestyle choices alone do not account for the increase in complex and chronic conditions. Other factors include: the globalisation of the workforce with frequent travel, less rest and increasing stress; socioeconomic influences on health; higher survival rates for diseases such as cancer and human immunodeficiency virus; an increase in obesity; and an increase in chronic and non-communicable diseases, such as hypertension, diabetes and chronic renal disease. The combined effect of an ageing population, lifestyle factors and health behaviours underpin the complexity of contemporary healthcare and nursing practice (Kuipers et al 2011).

These factors mean that nurses frequently provide care for patients with complex and chronic conditions, and often engage in multiple interactions with a patient over a long period of time. In some cases, these patients may be perceived by the nurse as exhibiting behaviour that is 'challenging' (Forrest 2012, Michaelsen 2012). To provide effective care, it is important for nurses to have a better understanding of the complexity associated with comorbid conditions and chronic illhealth, and the factors that can affect each episode of healthcare delivery.

\section{BOX 1. Definitions of commonly used terms in healthcare}

Chronic: a disease or disorder persisting for a long period, often for the remainder of a person's lifetime, or recurring frequently.

Chronicity: 'Consists of a fusion of identity with diagnosis... demoralising change of self from a person who has an illness to someone who is an illness or diagnosis' (Smith-Morris 2010).

Comorbidity: the simultaneous presence of two or more diseases in an individual which are associated with each other through pathogenetic mechanisms.

Complexity: the state of being complex, not simple, complicated, intractable, characterised by an interrelatedness of a multitude of components (Kannampallil et al 2011).

Empathy: 'Empathy doesn't require a clinician to feel vicariously what his or her patient feels; rather... it represents an emotional resonance and effort to understand that deepens one person's engagement with the experiences and suffering of another' (Aronson 2014).

Multimorbidity: the simultaneous presence of two or more diseases which appear randomly not having any connection to each other through pathogenetic mechanisms. It is 'the co-existence of multiple chronic conditions' typically resulting in the person presenting with complex needs and 'significant changes in... functional health and quality of life' (Sampalli et al 2012).

Person-centred care: 'A holistic approach to the planning, delivery and evaluation of healthcare that is grounded in mutually beneficial partnerships between healthcare professionals, patients and families. It is built on the understanding that patients bring their own experiences, skills and knowledge about their condition and illness; and that patient's perspectives (concerns, ideas and expectations) about their illness are as important as their physical examination and past medical history' (Levett-Jones 2014).

Therapeutic communication: 'Demonstrating care, genuine concern and empathy, and interest in the patient as a unique individual by actively listening (hearing, understanding and believing the patient)' (Levett-Jones 2014). 


\author{
KEY POINT \\ It can be beneficial to \\ identify the medical, \\ systemic, situational and \\ personal factors that may \\ contribute to the challenges \\ of working effectively with \\ a patient. This can improve \\ the nurse's understanding \\ of the complexity of the \\ patient's circumstances
}

There are significant differences in providing care to patients with a single episode of illness, for example an uncomplicated appendectomy, and providing care to patients with complex and chronic conditions, which may include comorbidities, chronic pain and socioeconomic issues (Nolte et al 2008). Additional education and skills development are necessary to prepare healthcare teams and nurses to meet these challenges (World Health Organization (WHO) 2010, Kuipers et al 2014).

\section{TIME OUT 1}

Consider a patient you have encountered in your nursing practice who has chronic comorbid conditions, whereby the complexity of the situation and their healthcare needs have affected your efforts to develop a therapeutic relationship. Answer the following questions:

"What illnesses does the person live with (comorbidities)?

" How long has the person had their illness or illnesses? How does the illness affect the person's life (chronicity)?

»What is the person's level of knowledge about their illnesses and health status (health literacy)?

"What family and carer support is available?
"What socioeconomic issues does the person experience?

"What access to health services does the person have? Are they geographically isolated?

\section{Factors associated with healthcare complexity}

A range of factors may influence the complexity of an increasing number of healthcare presentations, including: comorbid conditions, age, frailty, socioeconomic issues, culture, environment, behaviour and systemic factors (Figure 1) (Kuipers et al 2011).

\section{TIME OUT 2}

Consider the clinical scenario in Time out 1 and compare your notes with the information about healthcare complexity in Figure 1. Can you identify other factors that might affect the care the patient is receiving? What systemic and situational factors are contributing to the complexity of the patient's presentation?

It can be beneficial to identify the medical, systemic, situational and personal factors that may contribute to the challenges of working effectively with a patient. This can improve the nurse's understanding of the complexity of the patient's circumstances. Typically, the patient will have a need for person-centred healthcare and social

\section{Figure I. Factors associated with healthcare complexity}

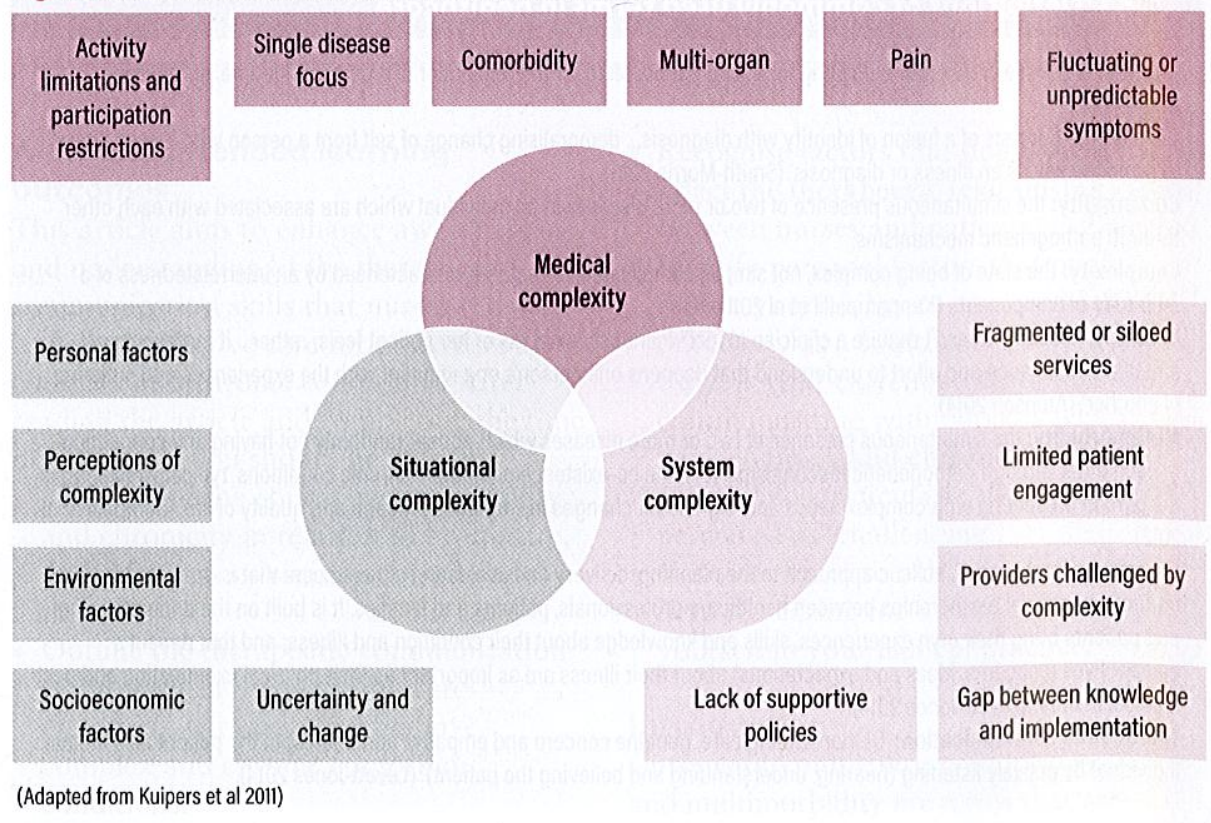


services that are delivered with empathy and compassion. However, healthcare providers might assume that the person will coordinate and manage many aspects of their healthcare needs themselves. In such situations, tensions may arise between the needs of the patient and the expectations of healthcare providers, which may adversely affect care and health outcomes. Research suggests that the effectiveness of care for patients with complex and chronic conditions is strongly affected by the quality of communication, the therapeutic relationship, and the rapport between the healthcare professional and the patient, as well as others involved in their care and support (Checton et al 2012).

\section{Effective communication}

Well-developed therapeutic communication skills are required for nurses to navigate complex and difficult circumstances successfully. It is important for nurses to be able to solve problems, manage busy workloads and use a range of specialist knowledge in their clinical practice. Technical competence and practical skills, for example learning to manage a patient with a central venous catheter or becoming competent in the use of the latest version of computerised equipment, have become an important aspect of daily nursing practice. As a result, the role that communication has in effective nursing care might be overlooked, with nurses focusing on these technical and practical tasks. It is important that nurses continue to prioritise person-centred care in complex and challenging circumstances.

\section{TIME OUT 3}

List the competencies related to communication and interpersonal skills that are expected of all registered nurses. Once you have done this, review the communication and interpersonal skills on page 9 of the Standards for Competence for Registered Nurses (Nursing and Midwifery Council (NMC) 2014) and indicate the areas that you think are particularly relevant to working with people with complex and chronic healthcare needs.

Communication and interpersonal skills are emphasised as one of the four main areas of professional nursing practice (NMC 2014). Communication errors have been implicated in up to $70 \%$ of sentinel events (unanticipated events resulting in death or serious physical or psychological injury, not related to the natural course of the patient's illness) in healthcare settings (Leonard et al 2004), with the potential for error increasing as levels of complexity rise. Thus, there is an increasing emphasis on the importance of effective communication skills in nursing, from undergraduate level onwards, on the basis that all nurses are likely to be involved in the care of patients with chronic conditions (Ghebrehiwet 2008, 2010).

Several international organisations have developed competencies and guidelines for chronic disease practice (WHO 2005, National Association of Chronic Disease Directors 2013). Box 2 shows the communication skills that are essential in the care of patients with complex and chronic conditions.

Communication skills may vary depending on the setting and situation. For example, nursing in acute and emergency care settings requires the ability to take charge and act quickly. By contrast, it is necessary for nurses in complex and chronic care to collaborate closely with the patient, their family and support networks, and to maintain effective relationships

BOX 2. Essential communication skills required in complex and chronic care

Healthcare professionals should:

» Provide patient-centred care that includes communicating effectively, supporting changes in health-related behaviours and assisting self-management.

"Use therapeutic principles to engage, maintain and, where appropriate, disengage from professional caring relationships, and ensure professional boundaries are respected.

"Work collaboratively with patients, families, other healthcare providers and communities, and members of the multidisciplinary team to provide coordinated treatments and support across the care continuum.

" Develop educational resources and provide educational sessions that support and enhance individual, family and community health literacy.

» Establish effective working relationships with other programmes, government agencies and non-governmental groups to promote support for chronic disease prevention and management.

" Be involved in designing and using patient registries, using computer technologies and communicating online with patients.

" Maintain accurate, clear and complete patient records, including the use of electronic formats, using appropriate and plain language.

" Develop and implement evidence-based interventions and conduct evaluations to ensure ongoing communication and feedback regarding the effectiveness of such interventions.

(Adapted from World Health Organization 2005, International Council of Nurses 2010, National Association of Chronic Disease Directors 2013, Nursing and Midwifery Council 2014) 


\author{
KEY POINT \\ The challenge is not \\ the patient themselves, \\ but the relationship \\ between the nurse and \\ the patient. Breakdowns \\ in communication and \\ therapeutic relationships \\ between healthcare \\ professionals and patients \\ are not limited to nursing
}

with the multidisciplinary team over long periods of time. Effective communication and interpersonal skills are necessary to maintain therapeutic relationships over time. Caring for a patient whose recurring symptoms and repeated presentations to healthcare services are characterised by chronicity and complexity could be challenging for healthcare professionals, regardless of their level of skills and experience.

\section{Supporting patients with complex needs}

For more than a decade, one of the authors (RR) practised as a clinician, providing support and counselling for people with rare chronic autoimmune diseases. This role provided an opportunity to work closely with people experiencing illnesses that affected every aspect of their lives, and offered a chance to observe the challenges associated with chronic conditions and the uncertainty and variability of complex multisystem illnesses. During those years, it was not uncommon for nursing colleagues to ask RR: 'How do you cope with working with people who don't get better?' This question seemed to imply past experiences of difficulty and perhaps a sense of futility.

\section{TIME OUT 4}

Consider the clinical scenario in Time out 1 and answer the following questions:

"What is it about trying to develop an effective therapeutic relationship with this person that you find difficult?

" Are there particular communication difficulties that you can identify?

"Can you identify the emotions that you experience when you see this person?

" How does this person challenge your expectations of yourself as a nurse?

"What measures do you take to manage your responses?

" Do you have any experience of chronic illness that could influence your expectations of how this person should behave?

Research that has explored the concept of 'difficult' patients disputes the commonly held notion that the person themselves is challenging or complex (Michaelsen 2012). Nurses may feel surprised at hearing comments from other healthcare professionals, caregivers or family about how a particular patient is 'challenging' or 'difficult', yet they have not experienced this in their interactions with the patient. Michaelsen (2012) suggested that "there is no unequivocal definition of a "difficult" patient'. The challenge is not the patient themselves, but the relationship between the nurse and the patient. Breakdowns in communication and therapeutic relationships between healthcare professionals and patients are not limited to nursing. Blackall and Green (2012) asserted that where disruptions occur between doctors and patients 'the genesis of most difficult behaviour lies in the doctor-patient relationship'.

Responding to patients in a professional manner that demonstrates compassion and empathy, despite difficult relationships, requires self-awareness and a personal understanding of the specific behaviours that the nurse may find demanding. Working closely with people who are experiencing painful and challenging circumstances in their lives will often evoke an emotional response. A significant component of nursing practice has been described as emotional labour. In contrast with routine physical care and task-based activities, emotional labour can often involve conflicting emotional responses. With increasing emotional intensity, distancing one's self physically and emotionally from the patient is understandable. However, emotional distancing has a negative effect on the quality of care provided, with an increased likelihood that social and health-related problems might be neglected (Michaelsen 2012).

At this point, it is essential for nurses to reflect on what is happening. Forrest (2012) stated that 'as nurses it is important that we are able to identify personal factors and emotions and separate them from the patient's problem to address his or her needs effectively and sympathetically'. There are various behaviours that can 
influence healthcare professionals' perceptions of the person being cared for. The effect of these will differ depending on factors such as personality, fatigue, stress, and any personal commitments and challenges experienced outside of work. However, acknowledging the role of one's responses, both verbal and non-verbal, in difficult interactions requires the capacity to reflect on one's communication. The Institute for Healthcare Communication (2011) stated that 'extensive research has shown that no matter how knowledgeable a clinician might be, if he or she is not able to open good communication with the patient, he or she may be of no help'.

\section{TIME OUT 5}

Identify which of the behaviours below have been problematic or have led you to label a patient as 'difficult', 'challenging' or 'demanding':

1. The patient:

"Does not adhere to medical advice

"Does not adhere to treatment

»Does not follow advice on self-care

2. The patient responds to your attempts to help with:

" Anger (threatening, bullying or patronising)

" Fear

" Rejection

» Further demands

\section{The patient:}

" Returns repeatedly with unexplained symptoms

" Attends multiple appointments but does not take any positive action

" Threatens complaints or litigation

4. Despite extensive treatment over a long period of time, there is no resolution of the patient's condition, for example non-healing wounds or persistent hyperglycaemia

List the emotions that you experienced in response to each of these behaviours. What strategies do you currently use to manage your emotional response and communication with patients? What could you do differently and what would be a more effective response?

\section{What you say, and how you say it, matters}

Providing effective healthcare for a patient whose behaviour is perceived as 'difficult' or 'challenging', and whose healthcare needs are complex and chronic, requires nurses to develop the ability to communicate effectively while acknowledging their own emotional responses, which may involve the urge to withdraw from the situation or speak harshly. Effective therapeutic communication begins with the nurse, who should work to strengthen personal attributes of authenticity and genuineness, empathy and self-awareness. A central feature of therapeutic communication is mindfulness, which is described as focused attention and awareness that enables the nurse to put aside any biases and assumptions, and use a non-judgemental approach in patient interactions. This creates an opportunity to be therapeutically present, which has been described as intentionally 'bringing one's whole self' into the interaction with the patient (Geller et al 2010). Being fully present enables the nurse to hear and validate the person's experience in such a way that the patient feels understood and able to trust the nurse to work collaboratively towards achieving the best possible outcomes (Rossiter et al 2014).

Listening to the patient's experience rather than focusing solely on their symptoms and adherence to treatment often reveals the competing difficulties they are having managing their illness, while trying to maintain normal functioning in their lives. The issues a patient with complex and chronic healthcare needs might be experiencing include: economic difficulties as a result of their illness; a sense of loss of control; and difficulty in understanding conflicting messages and advice from various healthcare professionals (Yen et al 2011). A further challenge is the 'invisibility' of many chronic conditions. Whereas a broken leg in a cast is a clear sign that a person has had an accident and is injured, by contrast, most patients with chronic conditions show no outward signs of their illness. Therefore, it can be difficult for others to recognise or acknowledge their needs.

Copen (2012) described the effect of 'words of cheer', such as 'you look so good'. For the patient, this may convey the message 'you can't really

\section{KEY POINT}

Listening to the patient's experience rather than focusing solely on

their symptoms and adherence to treatment often reveals the competing difficulties they are having managing their illness, while trying to maintain normal functioning in their lives. The issues a patient with complex and chronic healthcare needs might be experiencing include: economic difficulties as a result of their illness; a sense of loss of control; and difficulty

in understanding conflicting messages and advice from various healthcare professionals 
be sick' or 'you look fine to me', which invalidates the person's experience of illness. Conversely, comments such as 'don't give in, you need to fight this illness' may imply that the patient is not trying hard enough to recover from their condition, when in reality the patient is having difficulty in maintaining normal functioning. Healthcare professionals need to acknowledge the patient's experience of illness. Patient experiences and stories as told by patients are available from the Patient Voices website (www.patientvoices. org.uk/pilgrim.htm). The Patient Voices programme 'aims to facilitate the telling and the hearing of some of the unwritten and unspoken stories of ordinary people so that... clinicians directly involved in care may carry out their duties in a more informed and compassionate manner' (Pilgrim Projects Limited 2015).

\section{Caring for others and self-care}

A challenge for nurses is to deliver skilled and effective care with compassion and empathy while maintaining their own well-being (White 2014). It is often easier to provide advice about self-care to others while neglecting to care for oneself. The fundamental aspects of maintaining wellbeing include a healthy diet, adequate sleep and regular exercise.

It is important for nurses to ensure that they have a personal life outside of the profession, keeping in regular contact with friends and family. It can also be helpful to spend time exploring opportunities for reflection and quietness with practices such as yoga and mindfulness. White (2014) asserted that 'mindfulness is a significant concept for the discipline of nursing with practical applications for nurse well-being, the development and sustainability of therapeutic nurse qualities, and holistic health promotion'. Regular participation in individual or group clinical supervision can provide opportunities for reflection on practice, a non-judgemental space to explore the issues experienced during clinical encounters, to review personal ethics and moral values, and to enhance communication skills (Carroll 2011).

\section{Conclusion}

Nurses are encountering increasing numbers of patients with complex and chronic comorbid conditions. It is important to acknowledge that additional education and skills development might be necessary, to prepare nurses and other healthcare professionals to care for these patients. Effective communication is essential to foster and maintain therapeutic relationships and ensure high-quality care that meets the needs of this patient group in an empathetic manner. The ability of healthcare professionals, including nurses, to listen, develop rapport and trust, explain information and empathise is directly linked to patient satisfaction, and has been shown to have a significant effect on psychological and physical health outcomes. It is also vital that nurses consider their own health and well-being, to maintain their longevity in the profession.

\section{TIME OUT 6}

Now that you have completed the article, you might like to write a reflective account as part of your revalidation.

\section{References}

Aronson L (2014) Examining empathy. The Lancet. 384, 9937, 16-17.

Blackall GF, Green MJ (2012) "Difficult" patients or difficult relationships? The American Journal of Bioethics. 12, 5, 8-9.

Carroll M (2011) Supervision: A Journey of Lifelong Learning. In Shohet R (Ed) Supervision as Transformation: A Passion for Learning. Jessica Kingsley Publishers, London, 14-28.
Checton MG, Greene K, Magsamen-Conrad Ket al (2012) Patients' and partners' perspectives of chronic illness and its management. Families, Systems \& Health. $30,2,114-129$.

Copen L (2012) Talking to Someone with a Chronic Illness. edition.cnn.com/2012/09/11/ health/invisible-chronic-illness (Last accessed: 14 September 2016.)

Forrest C (2012) Working with 'difficult' patients. Primary Health Care. 22, 8, 20-22.

Geller SM, Greenberg LS, Watson JC (2010) Therapist and client perceptions of therapeutic presence: the development of a measure. Psychotherapy Research. 20,5, 599-610.

Ghebrehiwet T (2008) Core Competencies for Chronic Illness: A Paradigm Shift. Nurse Education on the Move. What about Educators' Competencies? 9-10 0ctober
2008, Plovdiv, Bulgaria. Presentation at the 7 th FINE Conference. www.fine-europe.eu/ wp-content/uploads/2014/09/Tesfamicael_ Ghebrehiwet_2.pdf (Last accessed: 14 September 2016.)

Ghebrehiwet T (2010) The Case for Focusing on Nurses Leading Chronic Diseases Control. Presentation at the launch of the ICN/Pfizer Global Chronic Diseases Initiative. June 24-25 2010, Washington, DC. www. c3health.org/wp-content/uploads/2010/08/ 
ICN-slides-NCDs-Tesfa.pdf (Last accessed: 14 September 2016.)

Institute for Healthcare Communication (2011) Impact of Communication in Healthcare. healthcarecomm.org/about-us/ impact-of-communication-in-healthcare (Last accessed: 14 September 2016.)

International Council of Nurses (2010) Delivery Quality, Servicing Communities: Nurses Leading Chronic Care. Internationa Council of Nurses, Geneva, Switzerland.

Kannampallil TG, Schauer GF, Cohen T et al (2011) Considering complexity in healthcare systems. Journal of Biomedical Informatics. $44,6,943-947$

Kuipers P, Ehrlich C, Brownie S (2014) Responding to health care complexity: suggestions for integrated and interprofessional workplace learning. Journal of Interprofessional Care. 28, 3, 246-248.

Kuipers P, Kendall E, Ehrlich C et al (2011) Complexity and Health Care: Health Practitioner Workforce Services, Roles, Skills and Training, to Respond to Patients with Complex Needs. www.health.qld.gov.au/ ahwac/docs/cet/complexcarefull1.pdf (Last accessed: 14 September 2016.)

Leonard M, Graham S, Bonacum D (2004) The human factor: the critical importance of effective teamwork and communication in providing safe care. Quality and Safety in Health Care. 13, Suppl 1, i85-i90.

Levett-Jones T (2014) Critical Conversations for Patient Safety: An Essential Guide for Health Professionals. Pearson Australia, Frenchs Forest NSW.

Michaelsen JJ (2012) Emotional distance to so-called difficult patients. Scandinavian Journal of Caring Sciences. 26, 1, 90-97.

Ministry of Health (2016) Health Loss in New Zealand: 1990-2013. www.health.govt.nz/ publication/health-loss-new-zealand-reportnew-zealand-burden-diseases-injuries-andrisk-factors-study-2006-2016 (Last accessed: 14 September 2016.

National Association of Chronic

Disease Directors (2013) Domains and Competencies. www.chronicdisease. org/?page=DomainsCompetencies (Last accessed: 14 September 2016.)
Nolte E KnaiC, McKee M (2008) Managing Chronic Conditions: Experience in Eight Countries. www.euro.who.int/ data/assets/ pdf file/0008/98414/E92058.pdf (Last accessed: 14 September 2016.

Nursing and Midwifery Council (2014) Standards for Competence for Registered Nurses. www.nmc.org.uk/globalassets/ sitedocuments/standards/nmc-standardsfor-competence-for-registered-nurses.pdf (Last accessed: 14 September 2016)

Pilgrim Projects Limited (2015) Patient Voices. www.patientvoices.org.uk/index.htm (Last accessed: 14 September 2016.)

\section{Rossiter R, Walton C, Scott R (2014) Key} Attributes of Therapeutic Communication. In Levett-Jones T (Ed) Critical Conversations for Patient Safety: An Essential Guide for Health Professionals. Pearson Australia, Frenchs Forest NSW, 102-112.

Sampalli T, Fox RA, Dickson R et al (2012) Proposed model of integrated care to improve health outcomes for individuals with multimorbidities. Patient Preference and Adherence. 6,757-764.
Smith-Morris C (2010) The Chronicity of Life the Acuteness of Diagnosis. In Manderson L, Smith-Morris C (Eds) Chronic Conditions, Fluid States: Chronicity and the Anthropology of Illness. Rutgers University Press, New Brunswick NJ, 21-37.

White L (2014) Mindfulness in nursing: an evolutionary concept analysis. Journal of Advanced Nursing. 70, 2, 282-294.

World Health Organization (2005) Preparing Health Care Workforce for the 21st Century: The Challenge of Chronic Conditions. www. who.int/chp/knowledge/publications/ workforce report.pdf (Last accessed: 14 September 2016.)

World Health Organization (2010) Framework for Action on Interprofessional Education and Collaborative Practice. apps.who.int/ iris/bitstream/10665/70185/1/WHO HRH HPN_10.3_eng.pdf?ua=1 (Last accessed: 14 September 2016.

Yen L, Gillespie J, Jeon YH et al (2011) Health professionals, patients and chronic illness policy: a qualitative study. Health Expectations. 14, 1, 10-20.

\section{NursingStandard}

\section{Open access}

Are you interested in making your article available to everyone, not just RCNi subscribers? RCNi Open Access enables authors to pre-pay and make the final version of their journal article freely available to all when it's published online. This service makes your article more discoverable and can increase its overall impact on nursing practice and patient care.
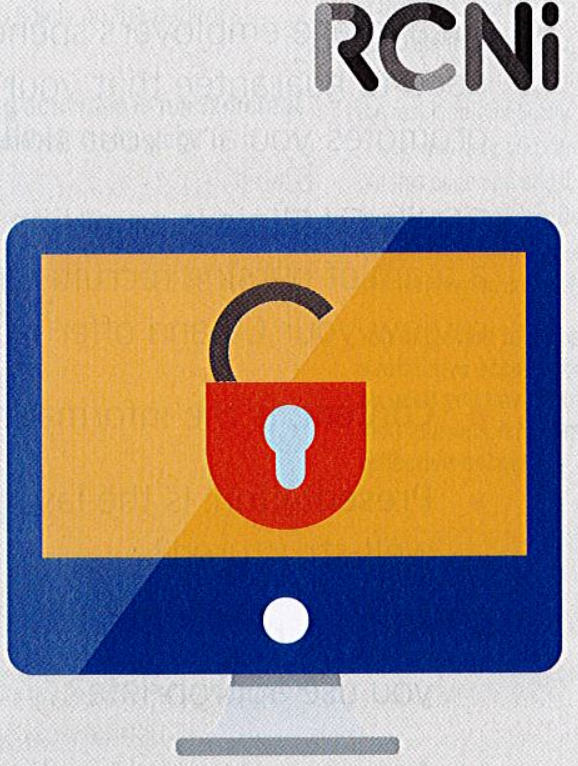

To find out more contact Evidence \& Practice editor Gwen Clarke gwen.clarke@reni.com 


\section{0 seconds to impress. Is your $\mathrm{CV}$ up to the job?}

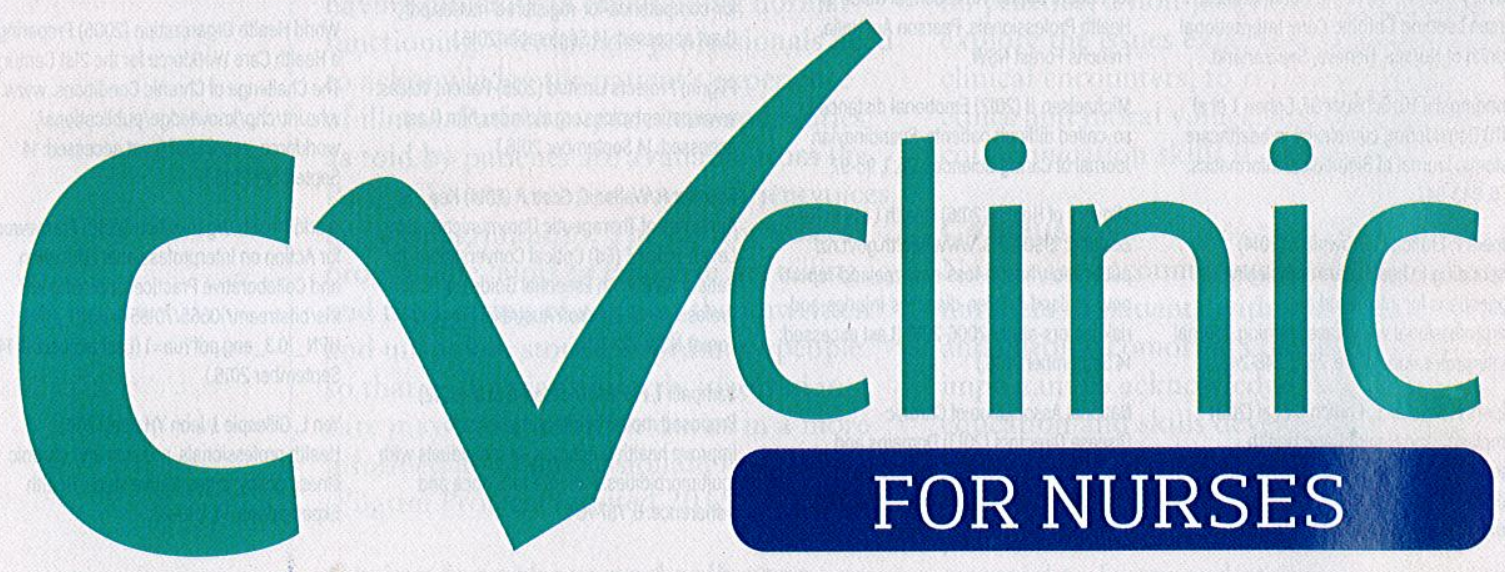

On average employers spend just 30 seconds looking at a CV, so you need to guarantee that your CV is articulate, attention-grabbing and promotes you and your skills in the most effective way possible.

RCNi's CV Clinic is an online careers advice service brought to you by a team of nursing recruitment specialists. For just $£ 25$, our team will review your CV and offer recommendations on:

- Content: Is the information relevant, interesting and coherent?

- Presentation: Is the layout of your CV visually appealing and well-structured?

- Style: Is the tone of your CV professional and is the language you use appropriate?

- Accuracy: Are the spelling and grammar consistently correct?

\section{Find out more at:} rcni.com/cv-clinic

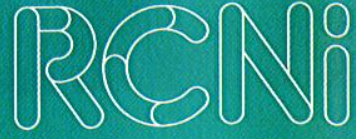

DIGITAL BRAND AE THE YEAR 


\section{Effective therapeutic communication}

TEST YOUR KNOWLEDGE BY COMPLETING SELF-ASSESSMENT QUESTIONNAIRE 864

1. Which statement is true?

a) The prevalence of chronic and complex conditions is increasing

b) The prevalence of chronic and complex conditions is decreasing

c) The prevalence of chronic and complex conditions remains the same

d) The terms 'chronic' and 'complex' are no longer used in healthcare

2. Multimorbidity is defined as:

a) Focused attention and awareness

b) The co-existence of multiple chronic conditions

c) Short-term treatment for severe injury or illness

d) A single long-term or recurring illness

3. What might increase the complexity of a patient's healthcare presentation?

a) Comorbid conditions

b) Socioeconomic issues

c) Systemic factors

d) All of the above

4. Communication errors have been implicated in which percentage of sentinel events?

a) Fewer than $30 \%$

b) Fewer than $40 \%$

c) Up to $60 \%$

d) Up to $70 \%$

5. To provide effective healthcare for patients who are perceived as having 'challenging' behaviour, nurses should:

a) Withdraw from the situation

b) Work to strengthen their authenticity, genuineness and self-awareness

c) Avoid behaviours that they find problematic

d) Physically and emotionally distance themselves from the patient

\section{A situational factor associated with healthcare} complexity is:
a) Lack of supportive policies
b) Limited patient engagement
c) Uncertainty and change
d) Fluctuating or unpredictable symptoms

7. When healthcare professionals find encounters with patients difficult, it is important they:

a) React in an abrupt manner

b) Disregard factors that might contribute to the patient's behaviour

c) Demonstrate compassion and empathy

d) Ignore the patient

8. Patients with chronic and complex healthcare needs may experience which of the following:

a) Economic difficulties as a result of their illness

b) A sense of loss of control

c) The 'invisibility' of their condition means that it can be difficult for others to respond to their needs

d) All of the above

9. Why may 'words of cheer' such as 'you look so good' be unhelpful for patients with chronic conditions?

a) They breach professional boundaries

b) They may invalidate the patient's experience of illness

c) They may improve the nurse-patient relationship

d) The nurse should focus solely on the patient's symptoms

10. Which of the following activities is fundamental for nurses to maintain their wellbeing?

a) An unhealthy diet

b) Lack of sleep

c) Regular exercise

d) Increased emotional labour

This self-assessment questionnaire was compiled by

Alex Bainbridge

The answers to this questionnaire will be published on 19 october

The answers to SAQ 862 on Using the British National Formulary (BNF), which appeared in the 21 September issue, are:

1.d 2. a 3.b 4.d 5. a 6.b 7.c 8.d 9. a 10.b
How to complete this assessment

This self-assessment questionnaire will help you to test your knowledge. It comprises ten multiple choice questions that are broadly linked to the article starting on page 54. There is one correct answer to each question.

"You can test your subject knowledge by attempting the questions before reading the article, and then go back over them to see if you would answer any differently.

"You might like to read the article before trying the questions. The correct answers will be published in Nursing Standard on 19 0ctober.

Subscribers making use of their RCNi Portfolio can complete this and other questionnaires online and save the result automatically.

Alternatively, you can cut out this page and add it to your professional portfolio. Don't forget to record the amount of time taken to complete it.

You may want to write a reflective account based on what you have learned. Visit journals.rcni.com/r/ reflective-account 